\title{
On the mean value of the two-term Dedekind sums
}

Kang Xiaoyu and Wu Zhengang*

${ }^{\text {*Correspondence: }}$ sky.wzgfff@163.com

Department of Mathematics, Northwest University, Xi'an, Shaanxi, P.R. China

\begin{abstract}
The main purpose of this paper is, using the properties of Gauss sums, the estimate for character sums and the analytic method, to study the mean value of the two-term Dedekind sums and give an interesting asymptotic formula for it.
\end{abstract}

MSC: $11 \mathrm{~F} 20 ; 11 \mathrm{~L} 40$

Keywords: two-term Dedekind sums; character sums; mean value; asymptotic formula

\section{Introduction}

Let $q$ be a natural number and $h$ be an integer prime to $q$. The classical Dedekind sums

$$
S(h, q)=\sum_{a=1}^{q}\left(\left(\frac{a}{q}\right)\right)\left(\left(\frac{a h}{q}\right)\right),
$$

where

$$
((x))= \begin{cases}x-[x]-\frac{1}{2}, & \text { if } x \text { is not an integer } \\ 0, & \text { if } x \text { is an integer }\end{cases}
$$

describes the behaviour of the logarithm of the eta-function (see $[1,2])$ under modular transformations. Many authors have studied the arithmetical properties of $S(h, q)$ and obtained many interesting results; see, for example, [3-5] and [6]. Now, for any odd prime $p$ and integer $m$ with $(m, p)=1$, we consider the mean value of the two-term Dedekind sums

$$
\sum_{a=1}^{p-1} \sum_{b=1}^{p-1} S\left(\left(a^{4}+m a^{2}\right) \cdot \overline{\left(b^{4}+m b^{2}\right)}, p\right)
$$

where $\bar{n}$ denotes the multiplicative inverse of $n \bmod p$, that is, $\bar{n} \cdot n \equiv 1 \bmod p$. For convenience, we provide $\bar{n}=0$ if $(n, p)>1$.

Here, we are concerned whether there exists an asymptotic formula for mean value (1). Regarding this problem, it seems that no one has studied it, at least we have not seen any related result before. The problem is interesting because it can reflect some properties of the value distribution of the polynomial Dedekind sums.

The main purpose of this paper is, using the properties of Gauss sums, the estimate for character sums and the analytic method, to study the mean value properties of the two-

\section{Springer}

(c)2013 Xiaoyu and Zhengang; licensee Springer. This is an Open Access article distributed under the terms of the Creative Commons Attribution License (http://creativecommons.org/licenses/by/2.0), which permits unrestricted use, distribution, and reproduction in any medium, provided the original work is properly cited. 
term Dedekind sums and give an interesting asymptotic formula for (1). That is, we shall prove the following theorem.

Theorem Let $p>3$ be an odd prime. Then, for any fixed integer $m$ with $(m, p)=1$, we have the asymptotic formula

$$
\sum_{a=1}^{p-1} \sum_{b=1}^{p-1} S\left(\left(a^{4}+m a^{2}\right) \cdot \overline{\left(b^{4}+m b^{2}\right)}, p\right)=\frac{1}{6} \cdot p^{2}+O\left(p^{\frac{3}{2}+\epsilon}\right)
$$

where $\epsilon$ denotes any fixed positive number.

Remark In this theorem, we only consider the special polynomial $x^{4}+m x^{2}$. However, we have not found an effective method to study the general polynomial $f(x)$. This problem should be a further study.

For general integer $q>3$, whether there exists an asymptotic formula for

$$
\sum_{\substack{a=1 \\(a, q)=1}}^{q} \sum_{\substack{b=1 \\(b, q)=1}}^{q} S\left(\left(a^{4}+m a^{2}\right) \cdot \overline{\left(b^{4}+m b^{2}\right)}, q\right)
$$

is an open problem, where we provide $\overline{\left(b^{4}+m b^{2}\right)}=0$ if $\left(b^{4}+m b^{2}, q\right)>1$.

\section{Several lemmas}

In this section, we shall give several lemmas, which are necessary in the proof of our theorem. Hereinafter, we shall use many properties of character sums and Gauss sums, all of these can be found in references [7] and [8]. First we have the following.

Lemma 1 Let $p$ be an odd prime, $\chi$ be any non-principal character $\bmod p$ with $\chi^{4} \neq \chi_{0}$, the principal character $\bmod p$. Then, for any integer $m$ with $(m, p)=1$, we have the identity

$$
\left|\sum_{a=1}^{p-1} \chi\left(a^{4}+m a^{2}\right)\right|^{2}=2 p+(-1)^{\frac{p-1}{2}}\left(\frac{m}{p}\right) \sum_{d=1}^{p-1} \chi(d) \sum_{a=1}^{p-1}\left(\frac{\left(a^{4}-d\right)\left(a^{2}-d\right)}{p}\right),
$$

where $\left(\frac{*}{p}\right)$ is the Legendre symbol.

Proof Since $\chi$ is a non-principal character mod $p$ with $\chi^{4} \neq \chi_{0}$, it must be a primitive character $\bmod p$, then from the properties of Gauss sums, we have

$$
\begin{aligned}
\sum_{a=1}^{p-1} \chi\left(a^{4}+m a^{2}\right) & =\frac{1}{\tau(\bar{\chi})} \sum_{a=1}^{p-1} \sum_{b=1}^{p-1} \bar{\chi}(b) e\left(\frac{b\left(a^{4}+m a^{2}\right)}{p}\right) \\
& =\frac{1}{\tau(\bar{\chi})} \sum_{a=1}^{p-1} \sum_{b=1}^{p-1} \bar{\chi}\left(b \bar{a}^{2}\right) e\left(\frac{b \bar{a}^{2}\left(a^{4}+m a^{2}\right)}{p}\right) \\
& =\frac{1}{\tau(\bar{\chi})} \sum_{b=1}^{p-1} \bar{\chi}(b) e\left(\frac{m b}{p}\right) \sum_{a=1}^{p-1} \chi\left(a^{2}\right) e\left(\frac{b a^{2}}{p}\right),
\end{aligned}
$$

where $\tau(\chi)=\sum_{a=1}^{p-1} \chi(a) e\left(\frac{a}{p}\right)$ denotes the classical Gauss sums, and $e(y)=e^{2 \pi i y}$. 
Xiaoyu and Zhengang Journal of Inequalities and Applications 2013, 2013:579

Page 3 of 7

http://www.journalofinequalitiesandapplications.com/content/2013/1/579

Note that the identities $|\tau(\chi)|=\sqrt{p}$ and

$$
\sum_{a=1}^{p-1} e\left(\frac{n a^{2}}{p}\right)= \begin{cases}p-1, & \text { if }(n, p)=p \\ \left(\frac{n}{p}\right) G(p)-1, & \text { if }(n, p)=1\end{cases}
$$

where

$$
G(p)=\sum_{a=0}^{p-1} e\left(\frac{a^{2}}{p}\right)=\sum_{a=1}^{p-1}\left(\frac{a}{p}\right) e\left(\frac{a}{p}\right)
$$

and

$$
G^{2}(p)=(-1)^{\frac{p-1}{2}} \cdot p
$$

From (2) and identities $\sum_{a=1}^{p-1} \chi^{2}(a)=\sum_{a=1}^{p-1} \chi^{4}(a)=0$, we have

$$
\begin{aligned}
& \left|\sum_{a=1}^{p-1} \chi\left(a^{4}+m a^{2}\right)\right|^{2} \\
& =\frac{1}{p}\left|\sum_{b=1}^{p-1} \bar{\chi}(b) e\left(\frac{m b}{p}\right) \sum_{a=1}^{p-1} \chi\left(a^{2}\right) e\left(\frac{b a^{2}}{p}\right)\right|^{2} \\
& =\frac{1}{p} \sum_{b=1}^{p-1} \sum_{d=1}^{p-1} \bar{\chi}(b \bar{d}) e\left(\frac{m b-m d}{p}\right) \sum_{a=1}^{p-1} \sum_{c=1}^{p-1} \chi\left(a^{2} \bar{c}^{2}\right) e\left(\frac{b a^{2}-c^{2} d}{p}\right) \\
& =\frac{1}{p} \sum_{b=1}^{p-1} \sum_{d=1}^{p-1} \chi(d) e\left(\frac{m b(1-d)}{p}\right) \sum_{a=1}^{p-1} \chi\left(a^{2}\right) \sum_{c=1}^{p-1} e\left(\frac{b c^{2}\left(a^{2}-d\right)}{p}\right) \\
& =\frac{1}{p} \sum_{b=1}^{p-1} \sum_{d=1}^{p-1} \chi(d) e\left(\frac{m b(1-d)}{p}\right) \sum_{\substack{a=1 \\
\left(a^{2}-d, p\right)=1}}^{p-1} \chi\left(a^{2}\right)\left(\left(\frac{b\left(a^{2}-d\right)}{p}\right) \cdot G(p)-1\right) \\
& +\frac{1}{p} \sum_{b=1}^{p-1} \sum_{d=1}^{p-1} \chi(d) e\left(\frac{m b(1-d)}{p}\right) \sum_{\substack{a=1 \\
a^{2} \equiv d \bmod p}}^{p-1} \chi\left(a^{2}\right)(p-1) \\
& =\frac{G(p)}{p} \sum_{d=1}^{p-1} \chi(d) \sum_{b=1}^{p-1}\left(\frac{b}{p}\right) e\left(\frac{m b(1-d)}{p}\right) \sum_{a=1}^{p-1} \chi\left(a^{2}\right)\left(\frac{a^{2}-d}{p}\right) \\
& +\sum_{d=1}^{p-1} \chi(d) \sum_{b=1}^{p-1} e\left(\frac{m b(1-d)}{p}\right) \sum_{\substack{a=1 \\
a^{2} \equiv d \bmod p}}^{p-1} \chi\left(a^{2}\right) \\
& -\frac{1}{p} \sum_{d=1}^{p-1} \chi(d) \sum_{b=1}^{p-1} e\left(\frac{m b(1-d)}{p}\right) \sum_{a=1}^{p-1} \chi\left(a^{2}\right) \\
& =(-1)^{\frac{p-1}{2}}\left(\frac{m}{p}\right) \sum_{d=1}^{p-1} \chi(d) \sum_{a=1}^{p-1} \chi\left(a^{2}\right)\left(\frac{(1-d)\left(a^{2}-d\right)}{p}\right)+2(p-1)
\end{aligned}
$$




$$
\begin{aligned}
& -\sum_{d=2}^{p-1} \chi(d) \sum_{\substack{a=1 \\
a^{2} \equiv d \bmod p}}^{p-1} \chi\left(a^{2}\right) \\
= & 2 p+(-1)^{\frac{p-1}{2}}\left(\frac{m}{p}\right) \sum_{d=1}^{p-1} \chi(d) \sum_{a=1}^{p-1} \chi\left(a^{2}\right)\left(\frac{(1-d)\left(a^{2}-d\right)}{p}\right)-\sum_{a=1}^{p-1} \chi\left(a^{4}\right) \\
= & 2 p+(-1)^{\frac{p-1}{2}}\left(\frac{m}{p}\right) \sum_{d=1}^{p-1} \chi(d) \sum_{a=1}^{p-1} \chi\left(a^{2}\right)\left(\frac{(1-d)\left(a^{2}-d\right)}{p}\right) \\
= & 2 p+(-1)^{\frac{p-1}{2}}\left(\frac{m}{p}\right) \sum_{d=1}^{p-1} \chi(d) \sum_{a=1}^{p-1}\left(\frac{\left(1-d \bar{a}^{2}\right)\left(a^{2}-d \bar{a}^{2}\right)}{p}\right) \\
= & 2 p+(-1)^{\frac{p-1}{2}}\left(\frac{m}{p}\right) \sum_{d=1}^{p-1} \chi(d) \sum_{a=1}^{p-1}\left(\frac{\left(a^{4}-d\right)\left(a^{2}-d\right)}{p}\right) .
\end{aligned}
$$

This proves Lemma 1.

Lemma 2 For any prime $p>3$, we have the estimate

$$
\left.\sum_{a=1}^{p-1}\left|\sum_{\substack{\chi \bmod p \\ \chi(-1)=-1}} \chi(a)\right| L(1, \chi)\right|^{2} \mid=O\left(p^{1+\epsilon}\right),
$$

where $\sum_{\chi(-1)=-1}$ mod denotes the summation over all characters $\chi \bmod p$ with $\chi(-1)=-1, \epsilon$ denotes any fixed positive number.

Proof See Lemma 5 of [9].

Lemma 3 Let $q>2$ be an integer, then for any integer a with $(a, q)=1$, we have the identity

$$
S(a, q)=\frac{1}{\pi^{2} q} \sum_{d \mid q} \frac{d^{2}}{\phi(d)} \sum_{\substack{\chi \bmod d \\ \chi(-1)=-1}} \chi(a)|L(1, \chi)|^{2},
$$

where $L(1, \chi)$ denotes the Dirichlet $L$-function corresponding to a character $\chi \bmod d$.

Proof See Lemma 2 of [6].

Lemma 4 Let $p>3$ be a prime. Then, for any integer $n$ with $(n, p)=1$, we have the identity

$$
\sum_{a=1}^{p}\left(\frac{a^{2}+n}{p}\right)=-1
$$

where $\left(\frac{*}{p}\right)$ denotes the Legendre symbol. 
Proof Since $\left(\frac{*}{p}\right) \equiv \chi_{2}^{0}$ is a primitive character mod $p$, so from the properties of Gauss sums $\tau\left(\chi_{2}^{0}\right)=\sum_{a=1}^{p-1} \chi_{2}^{0}(a) e\left(\frac{a}{p}\right)$, we know that

$$
\begin{aligned}
\sum_{a=1}^{p}\left(\frac{a^{2}+n}{p}\right) & =\frac{1}{\tau\left(\chi_{2}^{0}\right)} \sum_{a=1}^{p} \sum_{b=1}^{p-1}\left(\frac{b}{p}\right) e\left(\frac{b\left(a^{2}+n\right)}{p}\right) \\
& =\frac{1}{\tau\left(\chi_{2}^{0}\right)} \sum_{b=1}^{p-1}\left(\frac{b}{p}\right) e\left(\frac{n b}{p}\right) \sum_{a=1}^{p} e\left(\frac{b a^{2}}{p}\right) .
\end{aligned}
$$

Note that for any integer $u$ with $(u, p)=1$, we have

$$
\begin{aligned}
\sum_{a=1}^{p} e\left(\frac{u a^{2}}{p}\right) & =\left(\frac{u}{p}\right) \sum_{a=1}^{p} e\left(\frac{a^{2}}{p}\right) \\
& =\left(\frac{u}{p}\right) \sum_{a=1}^{p-1}\left(\frac{a}{p}\right) e\left(\frac{a}{p}\right) .
\end{aligned}
$$

Then, from (3) and (4), we have

$$
\begin{aligned}
\sum_{a=1}^{p}\left(\frac{a^{2}+n}{p}\right) & =\frac{1}{\tau\left(\chi_{2}^{0}\right)} \sum_{b=1}^{p-1}\left(\frac{b}{p}\right) e\left(\frac{n b}{p}\right)\left(\frac{b}{p}\right) \tau\left(\chi_{2}^{0}\right) \\
& =\frac{\tau\left(\chi_{2}^{0}\right)}{\tau\left(\chi_{2}^{0}\right)} \sum_{b=1}^{p-1} e\left(\frac{n b}{p}\right)=-\frac{\tau\left(\chi_{2}^{0}\right)}{\tau\left(\chi_{2}^{0}\right)}=-1,
\end{aligned}
$$

where we have used the fact that $\tau\left(\chi_{2}^{0}\right)=\sum_{a=1}^{p} e\left(\frac{a^{2}}{p}\right)$. This proves Lemma 4 .

\section{Proof of the theorem}

In this section, we shall complete the proof of our theorem. First, from the definition of $S(a, p)$, we have the computational formula

$$
S(1, p)=\sum_{a=1}^{p-1}\left(\frac{a}{p}-\frac{1}{2}\right)^{2}=\frac{(p-1)(p-2)}{12 p} .
$$

Then, from (5) and Lemma 3, we have

$$
\sum_{\substack{\chi \bmod p \\ \chi(-1)=-1}}|L(1, \chi)|^{2}=\frac{\pi^{2}}{12} \cdot \frac{(p-1)^{2}(p-2)}{p^{2}} .
$$

On the other hand, from Lemma 3 we also have

$$
S(a, p)=\frac{1}{\pi^{2}} \cdot \frac{p}{p-1} \cdot \sum_{\substack{\chi \bmod p \\ \chi(-1)=-1}} \chi(a)|L(1, \chi)|^{2} .
$$


If there exists a character $\chi_{1} \bmod p$ such that $\chi_{1}^{4}=\chi_{0}, \chi_{1}^{2} \neq \chi_{0}$ and $\chi_{1}(-1)=-1$, then from Weil's famous work (see [10]) we have the estimate

$$
\begin{aligned}
\left|\sum_{a=1}^{p-1} \chi_{1}\left(a^{4}+m a^{2}\right)\right|^{2} \cdot\left|L\left(1, \chi_{1}\right)\right|^{2} & =\left|\sum_{a=1}^{p-1} \chi_{1}\left(1+m \bar{a}^{2}\right)\right|^{2} \cdot\left|L\left(1, \chi_{1}\right)\right|^{2} \\
& =\left|\sum_{a=1}^{p-1} \chi_{1}\left(a^{2}+\bar{m}\right)\right|^{2} \cdot\left|L\left(1, \chi_{1}\right)\right|^{2} \ll p \cdot \ln ^{2} p .
\end{aligned}
$$

Then, from (7), (8) and Lemma 1, we have

$$
\begin{aligned}
& \sum_{a=1}^{p-1} \sum_{b=1}^{p-1} S\left(\left(a^{4}+m a^{2}\right) \cdot \overline{\left(b^{4}+m b^{2}\right)}, p\right) \\
& =\frac{1}{\pi^{2}} \cdot \frac{p}{p-1} \cdot \sum_{\substack{\chi \bmod p \\
\chi(-1)=-1}} \sum_{a=1}^{p-1} \chi\left(a^{4}+m a^{2}\right) \sum_{b=1}^{p-1} \bar{\chi}\left(b^{4}+m b^{2}\right) \cdot|L(1, \chi)|^{2} \\
& =\frac{1}{\pi^{2}} \cdot \frac{p}{p-1} \cdot \sum_{\substack{\chi \bmod p \\
\chi(-1)=-1}}\left|\sum_{a=1}^{p-1} \chi\left(a^{4}+m a^{2}\right)\right|^{2} \cdot|L(1, \chi)|^{2} \\
& =\frac{(-1)^{\frac{p-1}{2}}}{\pi^{2}} \frac{p}{p-1}\left(\frac{m}{p}\right) \sum_{d=1}^{p-1} \sum_{a=1}^{p-1}\left(\frac{\left(a^{4}-d\right)\left(a^{2}-d\right)}{p}\right) \sum_{\substack{\chi \bmod p \\
\chi(-1)=-1}} \chi(d) \cdot|L(1, \chi)|^{2} \\
& +\frac{1}{\pi^{2}} \cdot \frac{2 p^{2}}{p-1} \cdot \sum_{\substack{\chi \bmod p \\
\chi(-1)=-1}}|L(1, \chi)|^{2}+O\left(p \cdot \ln ^{2} p\right) \\
& =O\left(\left.\sum_{d=1}^{p-1}\left|\sum_{a=1}^{p-1}\left(\frac{\left(a^{4}-d\right)\left(a^{2}-d\right)}{p}\right)\right| \cdot\left|\sum_{\substack{\chi \bmod p \\
\chi(-1)=-1}} \chi(d) \cdot\right| L(1, \chi)\right|^{2} \mid\right) \\
& +\frac{1}{\pi^{2}} \cdot \frac{2 p^{2}}{p-1} \cdot \sum_{\substack{\chi \bmod p \\
\chi(-1)=-1}}|L(1, \chi)|^{2}+O\left(p \cdot \ln ^{2} p\right) .
\end{aligned}
$$

If $2 \leq d \leq p-1$, then from [10] we have the estimate

$$
\left|\sum_{a=1}^{p-1}\left(\frac{\left(a^{4}-d\right)\left(a^{2}-d\right)}{p}\right)\right| \ll \sqrt{p}
$$

From Lemma 4 we have

$$
\left|\sum_{a=1}^{p-1}\left(\frac{\left(a^{4}-1\right)\left(a^{2}-1\right)}{p}\right)\right|=\left|\sum_{a=0}^{p-1}\left(\frac{a^{2}+1}{p}\right)-1-2\left(\frac{2}{p}\right)\right| \ll 1 .
$$


From (10), (11) and Lemma 2, we have the estimate

$$
\begin{gathered}
\left.\sum_{d=1}^{p-1}\left|\sum_{a=1}^{p-1}\left(\frac{\left(a^{4}-d\right)\left(a^{2}-d\right)}{p}\right)\right| \cdot\left|\sum_{\substack{\chi \bmod p \\
\chi(-1)=-1}} \chi(d) \cdot\right| L(1, \chi)\right|^{2} \mid \\
\left.\ll \sqrt{p} \cdot \sum_{d=1}^{p-1}\left|\sum_{\substack{\chi \bmod p \\
\chi(-1)=-1}} \chi(d) \cdot\right| L(1, \chi)\right|^{2} \mid \ll p^{\frac{3}{2}+\epsilon} .
\end{gathered}
$$

Now, combining (6), (9) and (12), we deduce the asymptotic formula

$$
\sum_{a=1}^{p-1} \sum_{b=1}^{p-1} S\left(\left(a^{4}+m a^{2}\right) \cdot \overline{\left(b^{4}+m b^{2}\right)}, p\right)=\frac{1}{6} \cdot p^{2}+O\left(p^{\frac{3}{2}+\epsilon}\right)
$$

This completes the proof of our theorem.

\section{Competing interests}

The authors declare that they have no competing interests.

\section{Authors' contributions}

KX obtained the theorems and completed the proof. WZ corrected and improved the final version. All authors read and approved the final manuscript.

\section{Acknowledgements}

The authors express their gratitude to the referee for very helpful and detailed comments. This work is supported by the N.S.F. (11371291) and P.N.S.F. (2013JZ001) of P.R. China.

Received: 8 November 2013 Accepted: 21 November 2013 Published: 11 Dec 2013

\section{References}

1. Rademacher, H: On the transformation of $\log \eta(\tau)$. J. Indian Math. Soc. 19, 25-30 (1955)

2. Rademacher, H: Dedekind Sums. Carus Mathematical Monographs. Math. Assoc. of America, Washington (1972)

3. Apostol, TM: Modular Functions and Dirichlet Series in Number Theory. Springer, New York (1976)

4. Jia, C: On the mean value of Dedekind sums. J. Number Theory 87, 173-188 (2001)

5. Conrey, JB, Fransen, E, Klein, R, Scott, C: Mean values of Dedekind sums. J. Number Theory 56, $214-226$ (1996)

6. Zhang, W: On the mean values of Dedekind sums. J. Théor. Nr. Bordx. 8, 429-442 (1996)

7. Apostol, TM: Introduction to Analytic Number Theory. Springer, New York (1976)

8. Hua, LK: Introduction to Number Theory. Science Press, Beijing (1979)

9. Zhang, $W, Y i, Y, H e, X$ : On the $2 k$-th power mean of Dirichlet $L$-functions with the weight of general Kloosterman sums. J. Number Theory 84, 199-213 (2000)

10. Burgess, DA: On Dirichlet characters of polynomials. Proc. Lond. Math. Soc. 13, 537-548 (1963)

10.1186/1029-242X-2013-579

Cite this article as: Xiaoyu and Zhengang: On the mean value of the two-term Dedekind sums. Journal of Inequalities and Applications 2013, 2013:579 